\title{
DNA hypomethylation at the ZNF206-exon 5 CpG island associated with neuronal differentiation in mice and development of neuroblastoma in humans
}

\author{
HIROYUKI KAWASHIMA ${ }^{1,2}$, KIMINOBU SUGITO ${ }^{1}$, SHINSUKE YOSHIZAWA ${ }^{1}$, SHOTA UEKUSA ${ }^{1,2}$, \\ TAKESHI FURUYA ${ }^{1}$, TARO IKEDA ${ }^{1}$, TSUGUMICHI KOSHINAGA ${ }^{1}$, YUI SHINOJIMA ${ }^{2}$, RYO HASEGAWA ${ }^{2}$, \\ RAJEEV MISHRA ${ }^{2}$, JUN IGARASHI ${ }^{2}$, MAKOTO KIMURA $^{2}$, XIAOFEI WANG ${ }^{2}$, KYOKO FUJIWARA $^{2}$, \\ SRYMOYEE GOSH ${ }^{3}$ and HIROKI NAGASE ${ }^{2,4}$
}

\author{
Departments of ${ }^{1}$ Pediatric Surgery, and ${ }^{2}$ Cancer Genetics, Nihon University School of Medicine, \\ Tokyo, Japan; ${ }^{3}$ Department of Zoology, North-Eastern Hill University, Meghalaya, India; \\ ${ }^{4}$ Division of Cancer Genetics, Chiba Cancer Center Research Institute, Chiba, Japan
}

Received June 7, 2011; Accepted July 11, 2011

DOI: $10.3892 /$ ijo.2011.1234

\begin{abstract}
Differentiation of human neuroblastoma recapitulates neural crest development. In our whole genome DNA methylation screening of tissue-specific differentially methylated regions (T-DMRs) and developmental stage specific differentially methylated regions (DS-DMRs) we reported that the exon 5 CpG island (CpGi) of Zfp206 (human: ZNF206), which was required to maintain embryonic stem cells in a pluripotent state, was one of potent brain and testis-specific T-DMRs in mice. In this study methylation level of the CpG sites at Zfp206-exon 5 $\mathrm{CpGi}$ in mouse brain samples at three different developmental stages (15-day-old embryo; E15, new born; NB, 12-week adult; AD) were quantitatively analyzed and it was identified that Zfp206-exon 5 CpGi was the DS-DMRs in mouse brain. In AD brains, Zfp206-exon 5 CpGi was significantly hypomethylated and Zfp206 expression was repressed, compared with E15 and NB brains. Hence, mehtylation level of human 5'-end of $\mathrm{CpGi}$
\end{abstract}

Correspondence to: Dr Hiroki Nagase, Department of Cancer Genetics, Nihon University School of Medicine, 30-1 OhyaguchiKamimachi, Itabashi-ku, Tokyo 173-8610, Japan

E-mail: hnagase@chiba-cc.jp

Abbreviations: T-DMRs, tissue-specific differentially methylated regions; DS-DMRs, developmental stage differentially methylated regions; $\mathrm{CpGi}$, CpG island; E15, 15-day-old embryo; NB, new born; AD, 12-week adult; TrkA, tropomyosin receptor kinase A; NGF, nerve growth factor; hMC, homogeneous MassCLEAVE; MALDI-TOF MS, matrix-assisted laser desorption/ionization time-of-flight mass spectrometry; Tm, melting temperature; PCR, polymerase chain reaction; TBS, tris-buffered saline; PBS, phosphate-buffered saline; CTCF, CCCTC binding factor; UTR, untranslated region; AUF1, AU-rich element binding factor 1; HUR, Hu antigen R

Key words: DNA methylation, neuroblastoma, ZNF206, neuronal development at ZNF206-exon 5, which is homologous CpGi to mice, was analyzed in neuroblastomas. Although all four adrenal samples showed complete methylation at the homologous region, we found the hypomethylation in 7 out of 26 neuroblastomas and a significant association between the hypomethylation and poor prognosis. In neuroblastoma cell lines and specimens, the hypomethylation was also associated with ZNF206 expression. These data indicated that the changes in DNA methylation levels at the Zfp206-exon 5 might be one of the important factors during neuronal development in mice and that the hypomethylation of the homologous region induced ZNF206 expression in humans and was associated with human neuroblastomagenesis. Even though the function of ZNF206 and its expression regulation in neuroblastoma remain elusive, ZNF206 might be a candidate differentiation suppressor and prognosis marker in neuroblastoma.

\section{Introduction}

Epigenetic programing predetermines the developmental program and provides necessary direction for the multitude of changes that are required to proceed from a fertilized oocyte to a fully developed adult animal (1). It has been suggested that epigenetic changes are associated with development and differentiation. DNA methylation is one of the epigenetic factors and plays an important role in the diverse genomic process, such as gene regulation, chromosomal stability, parental imprinting and X-inactivation (2). Recent genome-wide DNA methylation searches indicate that $4-17 \%$ of $\mathrm{CpG}$ sites are different in methylation among tissues and developmental processes $(3,4)$. Tissue-specific differentially methylated regions (T-DMRs) and developmental-specific differentially methylated regions (DS-DMRs) are suggested to play important roles in development and differentiation (5). Therefore, disruption of epigenetic processes can lead to altered gene function and malignant cellular transformation (6). For example, Hox genes are involved in determining anterior-posterior embryonic pattern and govern 
the process of differentiation, and also they are related to cancers (7). It has been suggested that T-/DS-DMR is aberrantly methylated in cancer and may regulate tumor-suppressor genes and/or oncogenes.

Neuroblastoma cells originate from the postganglionic cells of the sympathetic nervous system and it is the most common extracranial solid tumor in childhood. Survival rate of the children with unfavorable neuroblastoma is still $<40 \%$, despite intensive multimodal therapy (8). Treatment of highrisk neuroblastoma with a differentiating agent 13-cis retinoic acid is beneficial for patients without progressive disease (9). Higher expressions of tropomyosin receptor kinase A (TrkA), nerve growth factor (NGF), which are neuronal differentiation and development factors, are important key factors and good prognosis markers in neuroblastomas $(9,10)$. The methylation levels at the T- and DS-DMRs, which also regulate tissue differentiation and development, may be aberrantly regulated in neuroblastomas.

The CpG sites at CpG island (CpGi) of the Zfp206-exon 5 (human: ZNF206-exon 5) are reported as a testicular and neuronal-specific DMRs and they are demethylated during late stage of mouse brain and testis development $(5,11)$. Here, we found that methylation state of the region associated with the ZNF206 expression and the homologous CpGi was aberrantly hypomethylated in human neuroblastomas, especially those with poor prognostic patients.

\section{Materials and methods}

Tissue samples. C57 BL/6J mice were purchased from Jackson Laboratory and maintained at Charles River Laboratories, Inc. (Yokohama, Japan). Brain specimens of the mice at three differential stages (15-day-old embryo; E15, new born; NB, 12-week adult; AD), were collected and stored as described previously (11).

Twenty-six primary neuroblastoma specimens from 1999 to 2007 were obtained from Nihon University Hospital at the time of diagnosis and they were analyzed under the approval of Nihon University Institutional Review Boards (IRB no. 51). Neither neoadjuvant chemotherapy nor irradiation therapy was given preoperatively to any of the patients. Four adrenal samples were collected from a nephroblastoma patient undergoing nephrectomy and from 3 neuroblastoma patients (cases 2, 15 and 26) undergoing tumor resections. A kidney sample for positive control of Western blotting was collected from a body donation. This specimen was analyzed under the approval of Nihon University Institutional Review Boards (no. 31) with the approved informed consent. All of the adrenal and kidney samples are pathologically normal. All of samples were immediately snap-frozen in liquid nitrogen and stored at $-80^{\circ} \mathrm{C}$ until use. Summary of these patients is shown in Table I.

Cell lines and culture condition. Human neuroblastoma cell lines, TN-1, NB9 and NB19 were obtained from Riken cell bank (Tsukuba, Japan) and CHP134, SK-N-SH and Kelly were obtained from American Type Culture Collection (Manassas, VA, USA). All of the human neuroblastoma cell lines were maintened in RPMI-1640 (Nakarai tesque, Kyoto,Japan) supplemented with 10 or $15 \%$ (NB9 and NB19) fetal bovine serum (Nichirei Bioscience, Tokyo, Japan), $100 \mathrm{IU} / \mathrm{ml}$ penicillin $\left(\mathrm{Gibco}^{\mathrm{TM}}\right.$, Carlsbad, CA) and $100 \mu \mathrm{l} / \mathrm{ml}$ streptomycin (Gibco). The cells
Table I. Summary of neuroblastoma cases

\begin{tabular}{|c|c|c|}
\hline No. of case & Period time (month) & Prognosis \\
\hline 1 & 36 & Alive \\
\hline 2 & 36 & Alive \\
\hline 3 & 36 & Alive \\
\hline 4 & 36 & Alive \\
\hline 5 & 34 & Dead \\
\hline 6 & 36 & Alive \\
\hline 7 & 36 & Alive \\
\hline 8 & 36 & Alive \\
\hline 9 & 36 & Alive \\
\hline 10 & 36 & Alive \\
\hline 11 & 36 & Alive \\
\hline 12 & 36 & Alive \\
\hline 13 & 36 & Alive \\
\hline 14 & 5 & Dead \\
\hline 15 & 6 & Alive with disease \\
\hline 16 & 4 & Dead \\
\hline 17 & 7 & Dead \\
\hline 18 & 36 & Alive \\
\hline 19 & 36 & Alive \\
\hline 20 & 26 & Alive \\
\hline 21 & 24 & Alive with disease \\
\hline 22 & 5 & Alive with disease \\
\hline 23 & 9 & Alive \\
\hline 24 & 11 & Alive \\
\hline 25 & 8 & Alive \\
\hline 26 & 5 & Alive \\
\hline
\end{tabular}

Summary of adrenal samples: Adrenal sample 1 was collected from nephroblastoma patient; Adrenal sample 2 was collected from case 2; Adrenal sample 3 was collected from case 15; Adrenal sample 4 was collected from case 26 .

were cultured in a $37^{\circ} \mathrm{C}$ humidified atmosphere containing $5 \%$ $\mathrm{CO}_{2}$ maintained in appropriate conditions recommended by the manufacturers.

DNA preparation and bisulfite treatment. Total genomic DNA was extracted from mouse brains, neuroblastoma specimens, neuroblastoma cells and adrenal samples with DNeasy tissue kit (Qiagen, Valencia, CA) and modified by sodium bisulfite with the EZ DNA methylation kit (Zymo Research, Orange, $\mathrm{CA})$. These methods were described in manufacturer's instructions.

Quantitative analysis of DNA methylation using base-specific cleavage and matrix-assisted laser desorption/ionization timeof-flight mass spectrometry (MALDI-TOF MS). Sequenom MassArray quantitative methylation analysis (12) using the Mass Array Compact System (www.sequenom.com) was employed for the quantitative DNA methylation analysis at $\mathrm{CpG}$ dinucleotides. This system utilizes mass spectrometry 
Table II. Primers and sequences.

\begin{tabular}{|c|c|c|}
\hline \multicolumn{2}{|l|}{ Primer name } & Sequences $\left(5^{\prime}-3^{\prime}\right)$ \\
\hline Mouse primer set & $\begin{array}{l}\text { Forward } \\
\text { Reverse }\end{array}$ & $\begin{array}{l}\text { aggaagagagGGAGAGGGGTTTATTTAGAATGT } \\
\text { cagtaatacgactcactatagggagaaggctTTACCACACTCAAAACACTCCTA }\end{array}$ \\
\hline Human primer set & & \\
\hline Primer set 1 & $\begin{array}{l}\text { Forward } \\
\text { Reverse }\end{array}$ & $\begin{array}{l}\text { aggaagagagGGAGGAGTGGGTTAGTAGG } \\
\text { cagtaatacgactcactatagggagaaggcAAAAATCAAAATAACTAAAAAC }\end{array}$ \\
\hline Primer set 2 & $\begin{array}{l}\text { Forward } \\
\text { Reverse }\end{array}$ & $\begin{array}{l}\text { aggaagagagTYGYGTAGATGAATTGTAATT } \\
\text { cagtaatacgactcactatagggagaaggctACCCCAAACAAAAAAAAATTTA }\end{array}$ \\
\hline Primer set 3 & $\begin{array}{l}\text { Forward } \\
\text { Reverse }\end{array}$ & $\begin{array}{l}\text { aggaagagagTTTTTGTTTTTGGYGTGG } \\
\text { cagtaatacgactcactatagggagaaggctCACCTAAACAAACACCTACTAACCC }\end{array}$ \\
\hline Primer set 4 & $\begin{array}{l}\text { Forward } \\
\text { Reverse }\end{array}$ & $\begin{array}{l}\text { aggaagagagGTATTGGGTGTAGTGGTGGG } \\
\text { cagtaatacgactcactatagggagaaggctAATCCACRCCAAAAACAA }\end{array}$ \\
\hline Primer set 5 & $\begin{array}{l}\text { Forward } \\
\text { Reverse }\end{array}$ & $\begin{array}{l}\text { aggaagagagGGGTTTYGTATGGGTAGTTAG } \\
\text { cagtaatacgactcactatagggagaaggctCAAACCCAAAATCTAACCC }\end{array}$ \\
\hline Primer set 6 & $\begin{array}{l}\text { Forward } \\
\text { Reverse }\end{array}$ & $\begin{array}{l}\text { aggaagagagTGGGAAGGGATATTTTTGTAG } \\
\text { cagtaatacgactcactatagggagaaggctACACCTAACTACCCATACRAAA }\end{array}$ \\
\hline
\end{tabular}

(MS) for the detection and quantitative analysis of DNA methylation using homogeneous MassCLEAVE (hMC) base-specific cleavage and MALDI-TOF MS (12). The METHPRIMER program (http://www.urogene.org/methprimer/index1.html) (13) was used to design bisulfite PCR primers (Table II). Each reverse primer has a T7-promoter tag for in vitro transcription (5'-cagtaatacgactcactatagggagaagget-3'), and the forward primer is tagged with a 10 mer to balance melting temperature (Tm) (5'-aggaagagag-3'). All primers were purchased from Operon (Tokyo, Japan). Polymerase chain reaction (PCR) amplification was performed using HotStar Taq Polymerase (Qiagen, Valencia, CA, USA) in a 5- $\mu 1$ reaction volume using PCR primers at $200 \mathrm{nM}$ final concentration and $1 \mu \mathrm{l}$ bisulfitetreated DNA $(\sim 20 \mathrm{ng} / \mathrm{ml})$ as a template. After the treatment with Shrimp alkaline phosphatase, $2 \mu \mathrm{l}$ of the PCR products was used as a template for in vitro transcription and RNase A cleavage for the T-reverse reaction ( 3 ' to either rCTP), as described in the manufacturer's instructions (Sequenom hMC). The samples were desalted and spotted on a 384-pad SpectroCHIP (Sequenom) using a MassArray nanodispenser (Samsung, Seoul, Korea), followed by spectral acquisition on a MassArray Analyzer Compact MALDI-TOF MS (Sequenom). The resultant methylation calls were analyzed by EpiTyper software v1.0 (Sequenom) to generate quantitative measurements for each $\mathrm{CpG}$ site or an aggregate of multiple $\mathrm{CpG}$ sites. Since Maldi-TOF mass-methylated peak does not denote a particular $\mathrm{CpG}$ site, but rather corresponds to the number of $\mathrm{CpG}$ sites methylated within the cleavage fragment, we decided to present average percent methylation of all $\mathrm{CpG}$ sites in the bisulfite PCR fragment with the standard curve.

Standard curve of quantitative DNA methylation analysis was performed by using $0,25,50,75$ and $100 \%$ methylated samples. BAC DNA (RPMI-11 341L6) obtained from Rosewell Park Cancer Institute, Buffalo, NY, USA was used as $0 \%$ methylation and M.Sss-1 double-treated BAC DNA was used as $100 \%$ methylation. The methylation reactions were carried out in 1X M.Sss1 buffer with $160 \mu \mathrm{M}$ SAM (New England Biolabs, Ipswich, MA, USA). In total reaction volume of $50 \mu 1,500 \mathrm{ng}$ BAC DNA was treated with $4 \mathrm{U}$ M.Sss1 for $1 \mathrm{~h}$ at $37^{\circ} \mathrm{C}$ and the reactions were stopped for $20 \mathrm{~min}$ at $65^{\circ} \mathrm{C}$ (14). This step was repeated twice. The standard curve was fitted and methylation levels were adjusted and quantified.

DNA methylation analysis of human neuroblastoma was performed as follows: At first, 3 primer sets were designed for analysis of human homologous region to mouse $\mathrm{CpGi}$ of ZNF206-exon 5 by 3 neuroblastoma specimens and adrenal samples, which were collected from three patients (cases 2, 15 and 26). As consequence, we decided the region that showed significant difference in methylation between neuroblastoma specimens and adrenal samples. Then, methylation levels of the region were analyzed in 26 neuroblastoma specimens, 4 adrenal samples and 7 neuroblastoma cell lines.

Western blotting analysis. Mouse brain specimens and neuroblastoma cells were collected and total cell lysates were prepared in M-PER mammalian protein extraction reagent (Thermo, Rockford, IL, USA) containing a protease-inhibitor cocktail (Nakarai tesque). Protein $(50 \mu \mathrm{g})$ was loaded on NuPAGE $^{+} 10 \%$ Bis-Tris gels (Invitrogen Life Technologies, Carlsbad, CA, USA) for electrophoresis. The proteins separated at $100 \mathrm{~mA}$ for $1 \mathrm{~h}$, then transferred to polyvinylidene difluoride membranes by using iblot transfer for 7 min (Invitrogen). For mouse brain specimens, the membranes were incubated with Tris-buffered saline (TBS), containing 5\% non-fat milk, $0.2 \%$ Tween-20 and a rabbit anti-ZNF206 polyclonal antibody (1:100) overnight (Abcam, Cambrigde, UK). For the neuroblastoma cells, the membranes were incubated with TBS, containing 5\% non-fat milk, $0.2 \%$ Tween-20 and a goat anti-ZNF206 polyclonal antibody (1:100) (R\&D systems, Mckinley, MN, USA) overnight in room temperature. The membranes were washed three times with a TBS containing $0.2 \%$ Tween-20. The immunocomplexed proteins were identified by reaction with a 
a)

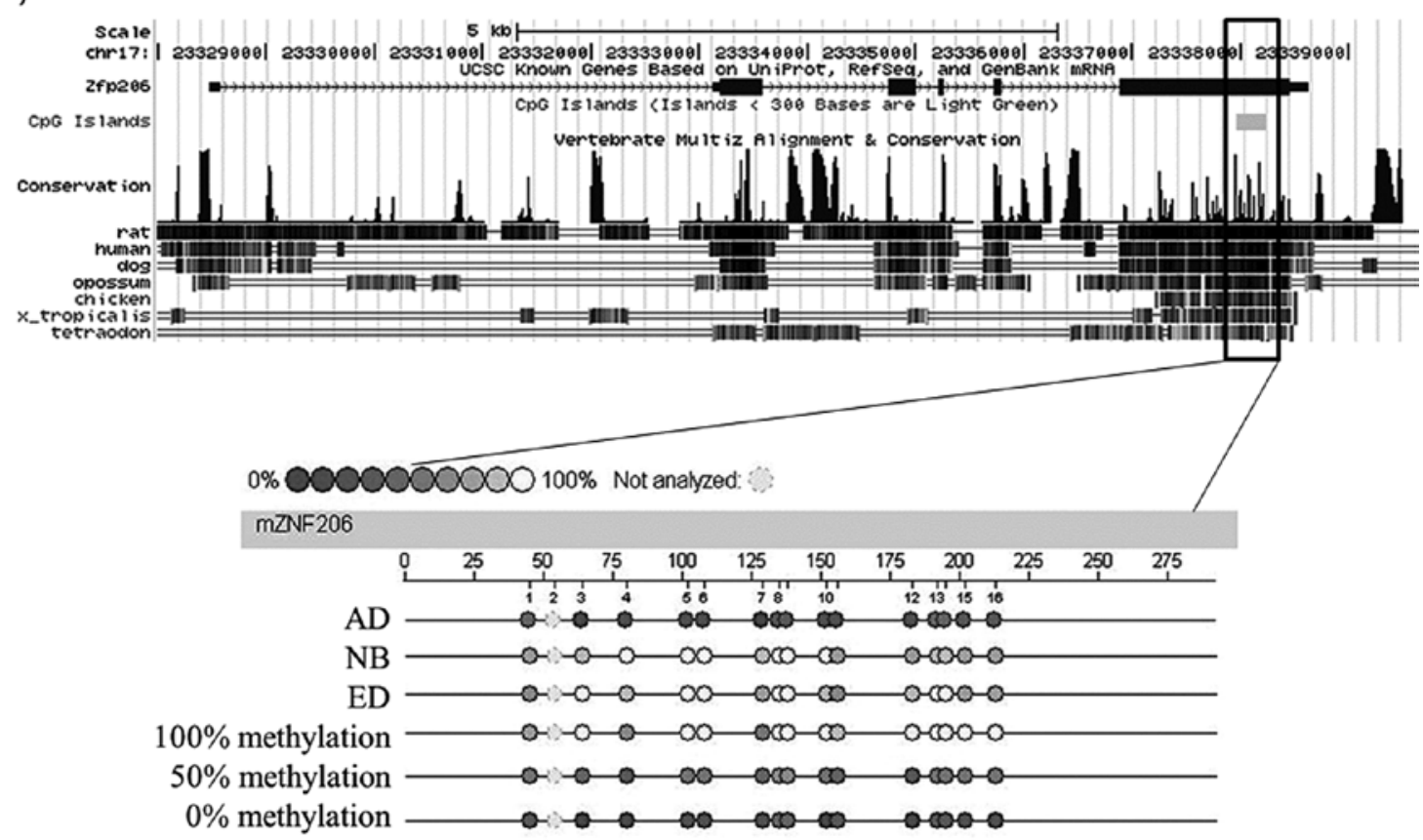

b)

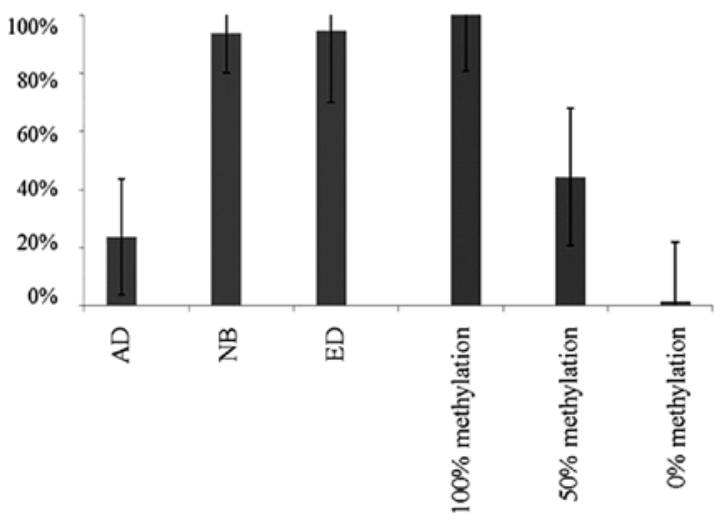

peroxidase-linked goat antibody to rabbit IgG (GE Healthcare UK Ltd., Little Chalfont, UK) for mouse brain specimens and the immunocomplexed proteins were identified by reaction with a peroxidase-linked horse antibody to goat $\operatorname{IgG}(\mathrm{R} \& \mathrm{D}$ systems) for neuroblastoma cells. Then these immunocomplexed proteins were detected by enhanced chemiluminescent reaction (Amersham Bioscience Inc., Piscataway, NJ, USA). Immunoblotting with antibody to actin (Abcam) provided an internal control for equal protein loading. Chemiluminescence was detected by LAS4000 (Fujifilm, Tokyo, Japan).

Immunohistochemical staining. In 17 neuroblastomas formalin-fixed, paraffin-embedded serial sections $(5 \mu \mathrm{m})$ were deparaffinized in xylene, rehydrated through graded alcohols, and washed with phosphate-buffered saline (PBS) for $15 \mathrm{~min}$. The sections were soaked in $10 \mathrm{mmol} / \mathrm{l}$ of sodium citrate buffer (pH 6.9) (Dako, Tokyo, Japan) and treated in a microwave for 15 min for antigen retrieval. Then the endogenous peroxidase activity was blocked with $3 \%$ hydrogen peroxidase in methanol for $30 \mathrm{~min}$, and non-specific staining was then blocked by incubation with normal goat serum (Nichirei) for $1 \mathrm{~h}$. The sections
Figure 1. The methylation level of CpGi at Zfp206-exon 5. (a) The mouse chr17:23337754-23338215 region is depicted based on the USCS database (Feb. 2006) (top). Inside the black square is the CpG site at Zfp206-exon 5 CpGi. Methylation levels of each mouse brain developmental stage and analyzed by using MassArray EpiTyper ${ }^{\circledR}$ method. Epigram (bottom) of each circle shows methylation levels of each $\mathrm{CpG}$ site representing a heat map created as quantitative measurement. (b) A bar graph shows average methylation levels of each sample. Adult brain is significantly hypomethylated in comparison with new born and E15 brains. ( $<<0.001$, Mann-Whitney U test).

Zff206

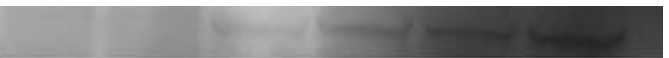

$\beta$-actin

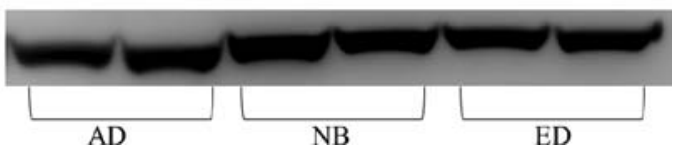

Figure 2. Western blotting of Zfp206 and $\beta$-actin. Zfp206 protein was detected in $\mathrm{NB}$ and $\mathrm{E} 15$ brain specimens, but not in $\mathrm{AD}$ brain.

were then incubated with $20 \mu \mathrm{g} / \mathrm{ml}$ ZNF206 antibody (R\&D systems) for $24 \mathrm{~h}$ at $4^{\circ} \mathrm{C}$. The sections were treated for $30 \mathrm{~min}$ at room temperature with goat secondary antibody against rabbit immunoglobulins (Nichirei). The sections were stained with AEC substrate kit (Vector Lab, Burlingame, CA, USA) at room temperature for $25 \mathrm{~min}$. After staining using the ACE substrate kit the sections were counterstained with hematoxylin for $1 \mathrm{~min}$.

Immunostaining was evaluated by the percentage of positive cells in tumor cells. Ten fields at $\mathrm{x} 400$ magnification were 


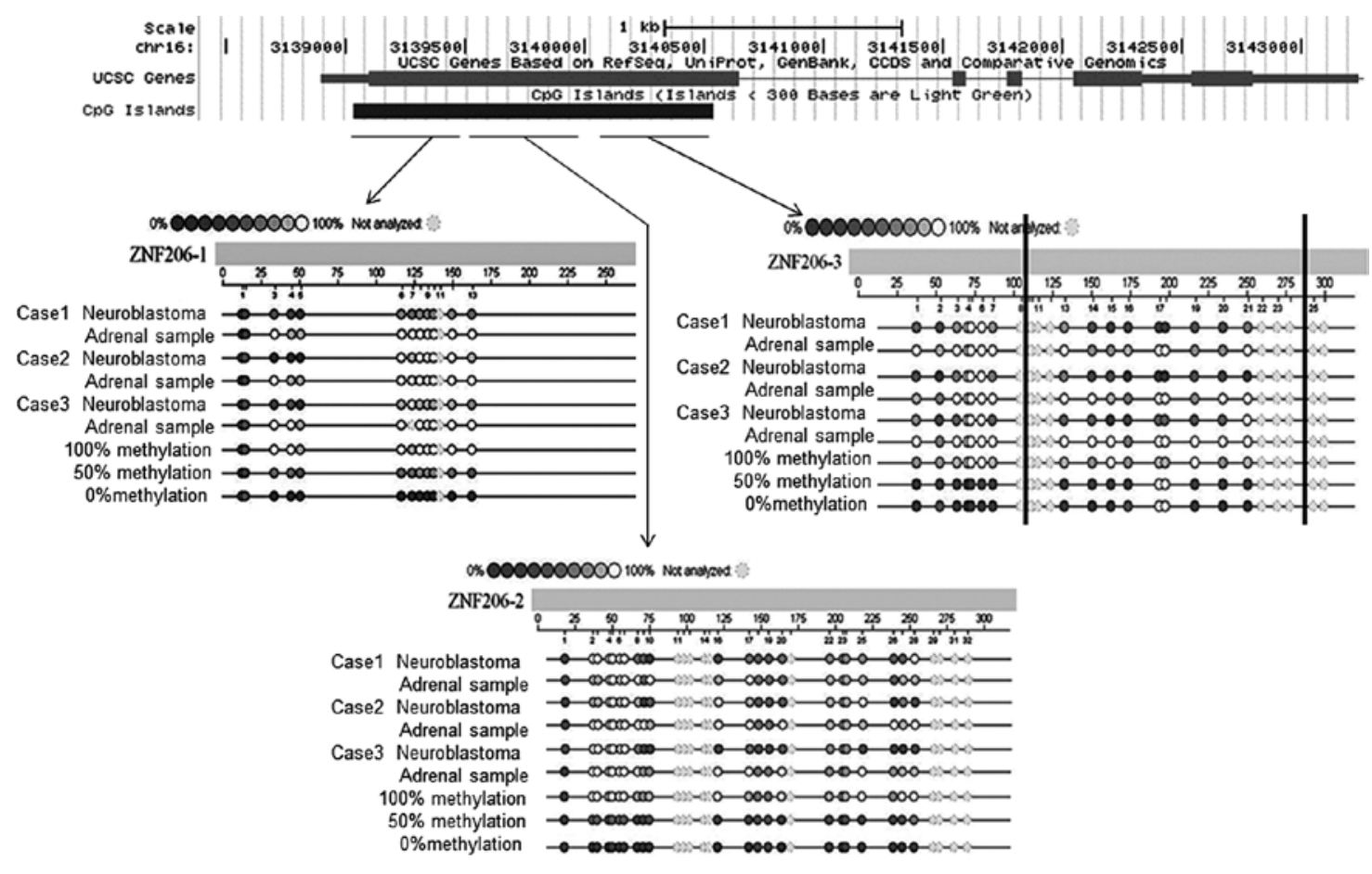

Figure 3. The methylation level of CpGi at exon 5 of ZNF206 in human neuroblastomas. Human homologous region to mouse CpGi at exon 5 of Zfp206 is shown (top). Note it is in an opposite direction of Fig. 1 in the mouse. Location of CpGi (chr16: 3079035-3080537) is at 5'-end of exon 5 to 3 ' untranslated region of ZNF206, based on the USCS database (Mar. 2006). (b) Quantitative methylation analysis of this CpGi by using MassArray EpiTyper method is depicted as a heat map of each CpG site. Three primer sets of ZNF206-1 (left), ZNF206-2 (middle) and ZNF206-3 (right) were designed for quantitative methylation analysis of CpGi located on ZNF206-exon 5. A significant difference in methylation at $\mathrm{CpG}$ sites between neuroblastoma specimens and adrenal samples in 3 neuroblastoma patients was clearly identified at CpGs of number 13-21 between black bars of ZNF206-3 located at 5'-end of ZNF206-exon 5 CpGi.

randomly selected from each sections and the number of positive tumor cells in whole field were calculated. Positive cells were defined as cells showing specific nuclear staining pattern and stronger staining in comparison with fibrous tissues.

Statistical analysis. For the statistical analysis, unpaired Mann-Whitney U test was performed by using SPSS software version 11.0.1 for Windows (SPSS GmbH Software, Munich Germany). For survival analysis the cut-off value was calculated. The methylation levels were pooled onto two groups by Youden index using 23 patients who had passed the 36-month observation period (15). The cut-off point between hyper and hypo-levels of DNA methylation at each candidate DMR was evaluated by ROC curve analysis. Survival curves were calculated according to Kaplan-Meier analysis and compared by a log-rank test. In all the statistical analysis differences were considered significant at $\mathrm{p}<0.05$.

\section{Results}

Methylation levels at CpG sites of Zfp206-exon 5 CpGi and its expression in mouse brain specimens. Methylation levels of the CpG site at Zfp206-exon $5 \mathrm{CpGi}$ in mouse brains were analyzed at three different developmental stages. This $\mathrm{CpG}$ site was estimated to be testicular and neuronal-specific DMRs and its methylation levels are low during late stage of brain and testis development in mice (Mice chr17:23337754-23338215 in the USCS database; Feb. 2006) (Fig. 1a). AD brain specimens were shown to be hypomethylated, compared with brain specimens of E15 and NB in these regions by using MassArray epityper method (Fig. 1b). In this experiment it was confirmed that the average methylation level of $\mathrm{CpG}$ site at Zfp206-exon $5 \mathrm{CpGi}$ in AD brain specimens were showing significantly and uniformly lower methylation levels, compared also with the other brain specimens (Fig. 1c). Western blotting revealed lower expression level of Zfp206 protein in AD brain specimens, compared with the other developmental stages (Fig. 2). Zfp206 protein expression status was correlated to methylation levels of $\mathrm{CpG}$ sites of the region.

Search for the greatest difference in somatic change within homologous ZNF206-exon 5 CpGi in human neuroblastoma. Analysis using the 3 primer sets designed to confirm methylation level at ZNF206-exon $5 \mathrm{CpGi}$ revealed that the 5'-end of CpGi at ZNF206-exon 5 showed the greatest difference in methylation between adrenal samples and neuroblastoma specimens among all CpG sites we analyzed (chr16:3,079,009-3,079,308, Mar, 2006 based on USCS database) in 3 neuroblastoma patients (Fig. 3). We decided that this region is a candidate marker site related to human neuroblastoma.

Methylation levels of candidate region in ZNF206 analyzed by using MassArray epityper method in 26 neuroblastoma specimens. All 4 adrenal samples were hypermethylated in the region. The average methylation level in 4 adrenal samples was $104.3 \pm 2.1 \%$. On the other hand, neuroblastoma hypermethylated and hypomethylated specimens showed intermediate methylation level and the average value of the candidate region 
a)
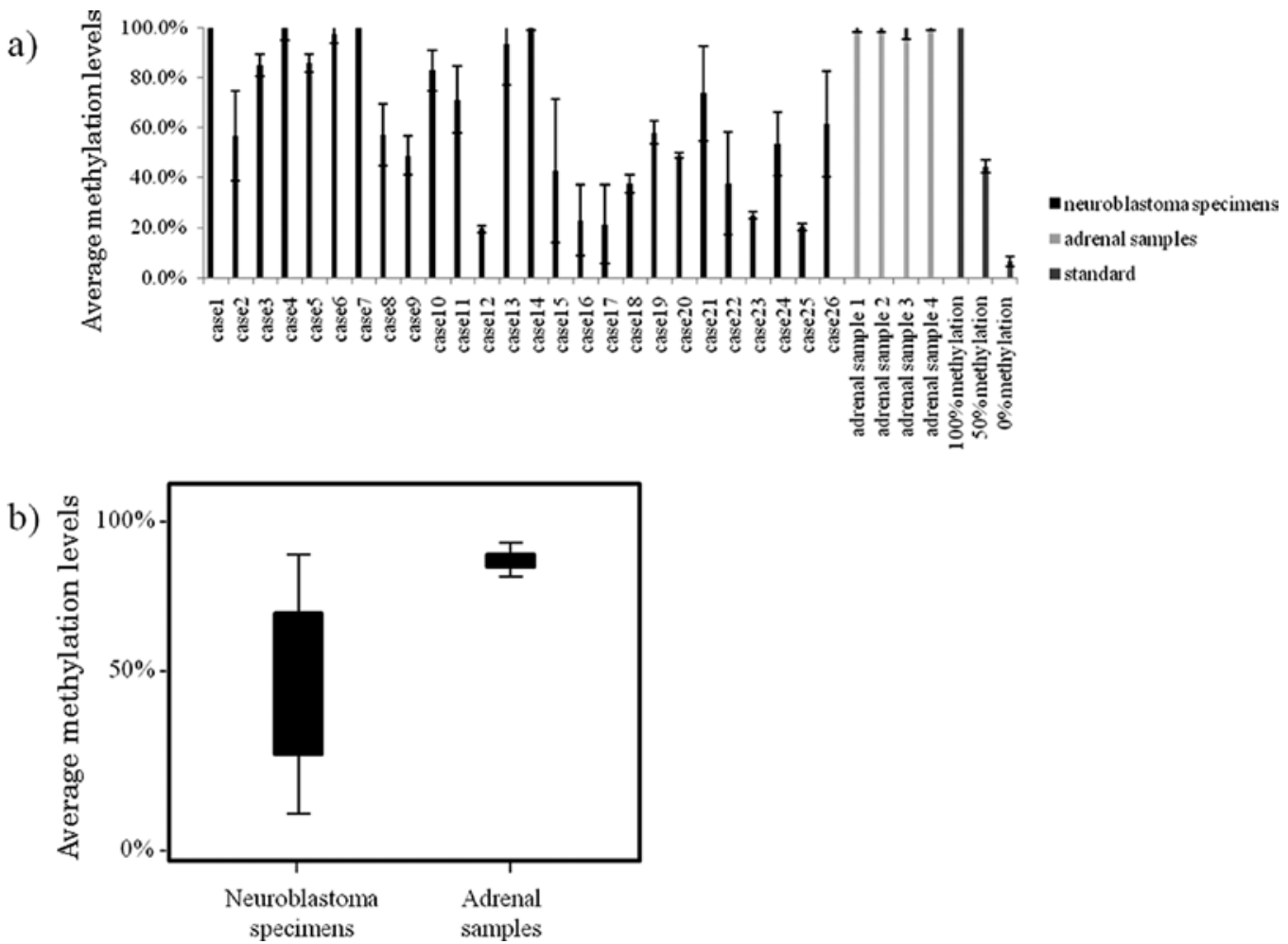

Figure 4. Methylation levels of the region in ZNF206-3. (a) Methylation levels of the region in ZNF206-3 were analyzed by using MassArray epityper method in 26 neuroblastoma specimens and 4 adrenal samples. The bar graph shows the average of methylation levels in the region indicated in Fig. 3. All 4 adrenal samples were hypermethylated. The average methylation level was $104.3 \pm 2.1 \%$. In neuroblastoma hypermethylated tumors and hypomethylated tumors were intermingled. (b) Variance of average methylation levels of neuroblastoma specimens is plotted as a box plot. Neuroblastoma specimens showed significantly lower methylation levels, compared with adrenal samples ( $\mathrm{p}=0.005$, Mann-Whitney $\mathrm{U}$ test).

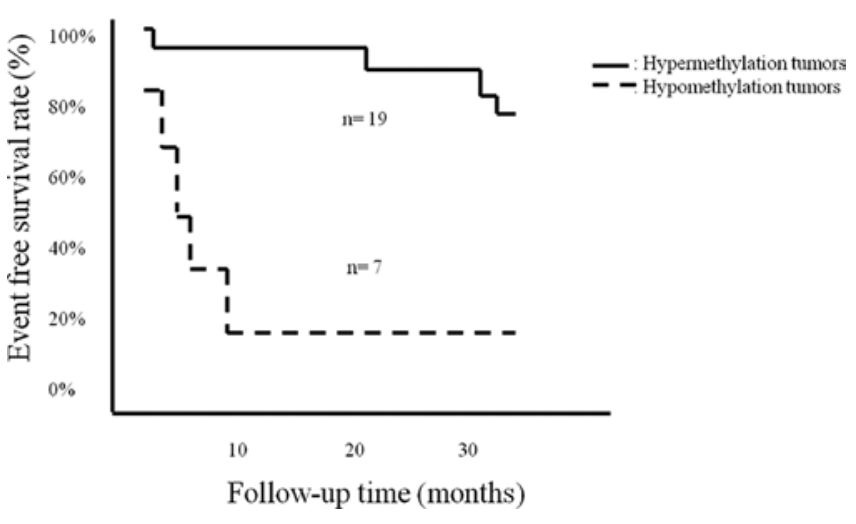

Figure 5. Survival analysis of neuroblastoma patients. For Kaplan-Meier analysis 26 neuroblastoma specimens were pooled into two groups (hypermethylation tumors: $>35.8 \%$, hypomethylation tumors: $\leq 35.8 \%$ ) by the cut-off value of ROC analysis measured by average methylation levels at the region of ZNF206-3. There was a significant correlation between hypermethylation and patient favorable outcome $(\mathrm{p}=0.001, \log$-rank test).

was $58.0 \pm 30.5 \%$. In 21 out of 26 neuroblastoma specimens, the methylation levels at candidate regions were low, compared with the average methylation level of candidate regions in 4 adrenal samples. The average of those in 26 neuroblastoma specimens was significantly lower, compared with that in the 4 adrenal samples ( $p=0.005$, Mann-Whitney U test) (Fig. 4).

For Kaplan-Meier analysis cut-off value of the methylation level at candidate region was calculated by using youden index and the methylation levels of 23 patients who had passed the observation period. The cut-off value was $35.8 \%$. Twenty-six neuroblastoma specimens were pooled into two groups depending on their methylation levels of the ZNF206-exon 5 regions (tumors with hypermethylation: $>35.8 \%$, tumors with hypomethylation: $>35.8 \%$ ) by the cut-off value. Nineteen out of 26 neuroblastoma specimens were in the hypermethylation tumor group. There was significant association between hypermethylation levels and patient outcome $(\mathrm{p}=0.001, \log$ rank test) (Fig. 5).

Methylation levels of the ZNF206-exon 5 regions and ZNF206 protein expression in neuroblastoma cells. All six neuroblastoma cell lines were categorized as hypermethylation of 40-95\% methylation level (Fig. 6a). In all six neuroblastoma cell lines ZNF206 protein expression, however, was repressed, compared with a positive control (a kidney specimen) (Fig. 6b).

ZNF206 staining in neuroblastoma specimens and adrenal samples. The stained tumor cells and non-stained tumor cells were intermingled in neuroblastoma section of case 24 (the methylation level: $22.7 \%$ ), which was a hypomethylated tumor. On the other hand, in neuroblastoma section of case 12 (methylation level: $55.2 \%$ ), which was a hypermethylated tumor the number of stained cells were less, compared with that of case 24 (Fig. 7a). Calculating \% positive cells in 17 neuroblastoma sections, a small number of cells from 5 out of 17 hypomethylated neuroblastoma sections expressed ZNF206. Moreover, the methylation levels of the region at ZNF206-exon $5 \mathrm{CpGi}$ 
a)

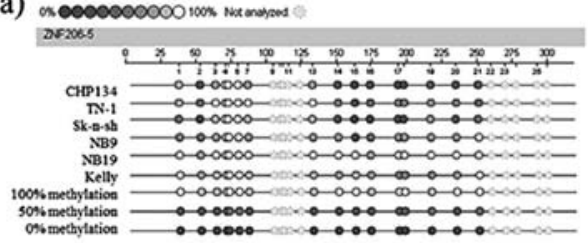

b)

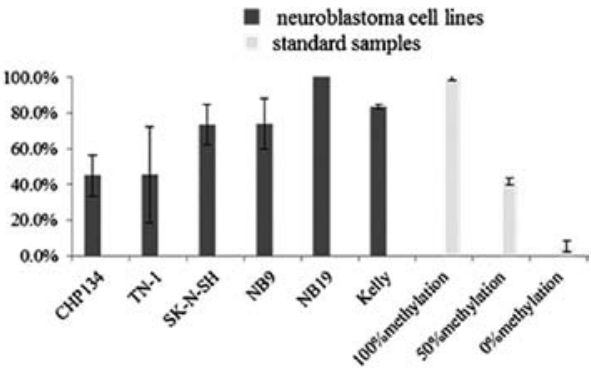

\section{Kidney CHP134 TN-1 SK-N-SH NB9 NB19 Kelly}

\section{ZNF206}

$\beta$-actin

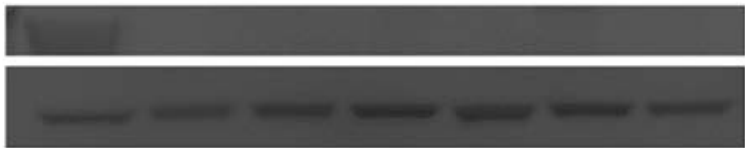

Figure 6. Methylation levels of the region of ZNF206-3 and ZNF206 expression in neuroblastoma cell lines. (a) Methylation level at each CpG in the region of ZNF206-3 is depicted as a circle by the heat map analyzed by using MassArray EpiTyper method in neuroblastoma cell lines. (b) Average methylation levels of CpGs in the region of ZNF206-3 in neuroblastoma cell lines are indicated in a bar graph. (c) Western blotting in human six neuroblastoma cell lines is shown. Repression of ZN206 compared with that of positive control (a kidney specimen) was seen in all neuroblastoma cell lines.

a)
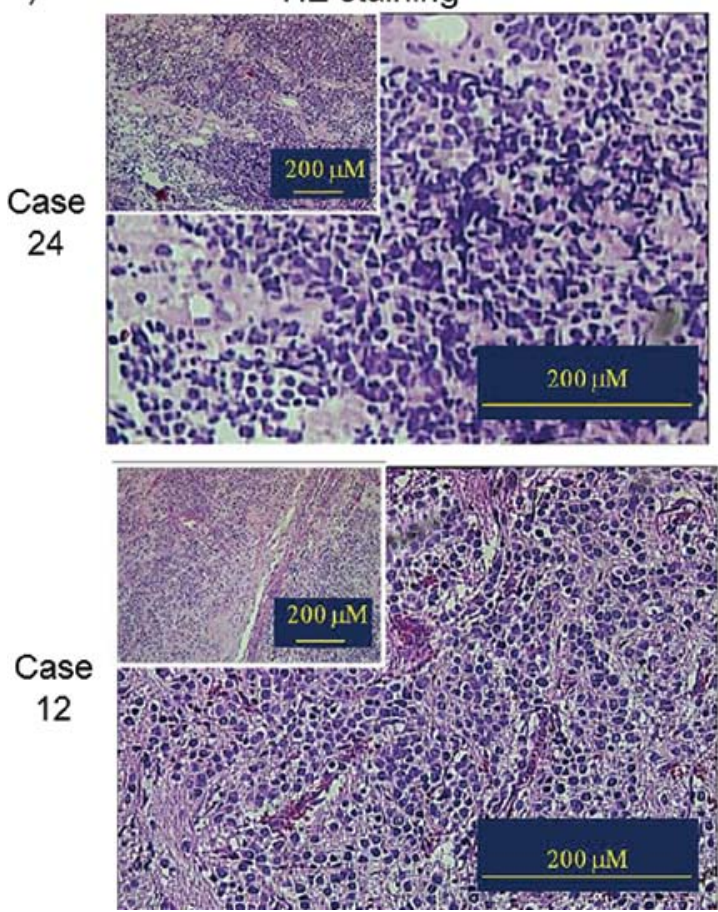

\section{ZNF206 staining}
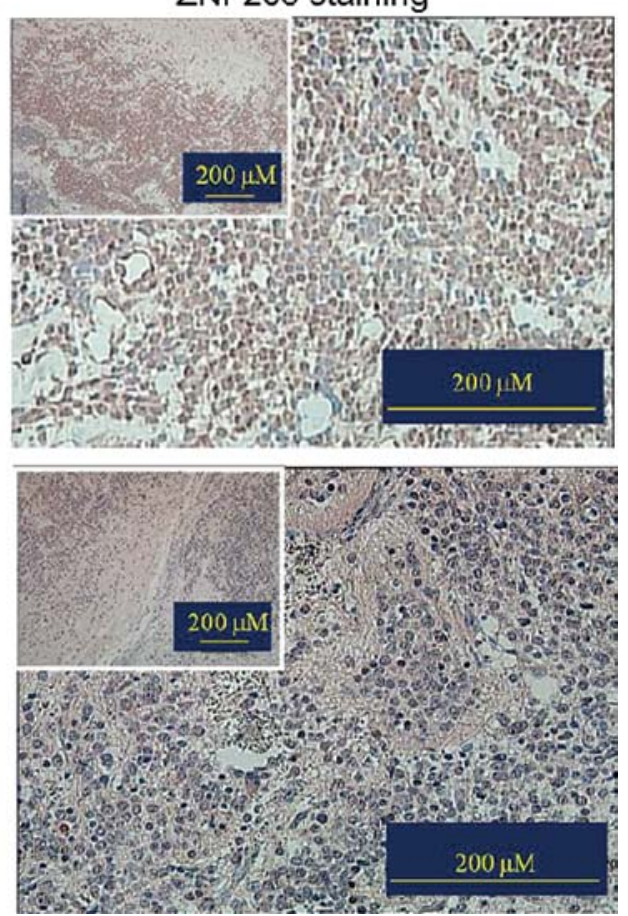

b) $100 \%$

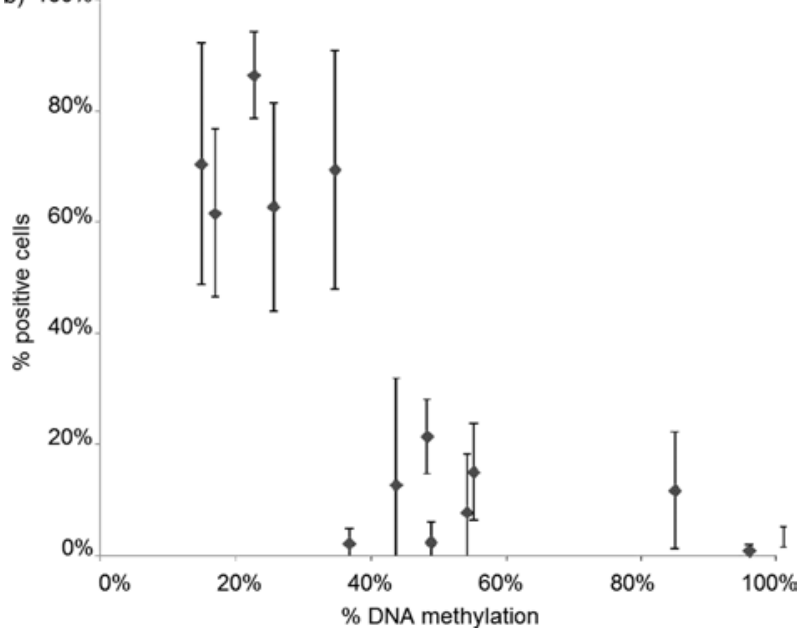

Figure 7. Immunohistochemical analysis demonstrating ZNF206. (a) ZNF206 expression in primary neuroblastoma sections were analyzed by immunohistochemical (IHC) staining. Upper figure shows the tumor section, which is a hypomethylated tumor (case 24 , methylation level of candidate region: $22.7 \%$ ) and lower figure shows section of a hypermethylated tumor (case 12 , methylation level of candidate region: 55.2\%). Positive cells were defined as tumor cells stained in nuclear area. The number of positive cells of case 12 was greater than those of case 24. (b) ZNF206 expression in 17 neuroblastoma sections was evaluated by IHC and indicated in the Y-axis. Average methylation level at ZNF206-3 of each sample is indicated in the X-axis. Five out of them were assigned as hypomethylated tumors and showed high percentage of positive cell staining. Twelve were assigned as hypermethylated tumors and showed low number of ZNF206 positive cells. Ten fields per section at x400 magnification were randomly selected and the number of positive tumor cells in whole field were counted. Percent positive cells were significantly correlated with methylation levels at the ZNF206-3 region $(\mathrm{p}=0.001$, Pearson correlation co-efficiency). 
were correlated with ZNF206 expression (Fig. 7b). Coincidently the cut-off of $35.8 \%$ in methylation showed a clear separation between high and low ZNF206expressed tumors, which also segregate between favorable and unfavorable prognosis.

\section{Discussion}

Many studies have shown that epigenetic abnormalities, especially alternation in DNA methylation, were involved in the development of various adult tumors $(16,17)$. In neuroblastoma, aberrantly methylated genes, $64 \%$ for $T H B S I ; 30 \%$ for TIMP-3; $27 \%$ for $M G M T ; 25 \%$ for $p 73 ; 18 \%$ for $R B 1 ; 14 \%$ for $D A P K$, p14ARF, p16INK4a and CASP8, and 0\% for TP53 and GSTPI have been reported and it is suggested that the striking differences in methylation status within neuroblastoma has revealed the existence of a methylator phenotype, which seems to be associated with more aggressive forms of neuroblastoma $(18,19)$. Although neuroblastoma development is associated with aberration of neural differentiation and DNA methylation, there were no reports showing a relationship between neuroblastoma and aberrant methylation at T-/DS-DMR, which play an important role in differentiation and development.

Zfp206 (human: ZNF206) encodes a zinc finger- and SCAN domain-containing protein, that functioned as a transcription factor and relate with differentiation in mouse embryonic stem cells, as a consequence of regulation on Oct4, Naolong and Sox 2 expression, which are master regulators of embryonic stem cells (20-23). Our present study showed that $\mathrm{CpG}$ sites at Zfp206-exon $5 \mathrm{CpGi}$ were differentially methylated during brain development and the methylation level was associated with Zfp206 expression. These results indicate that methylation level of CpG site at Zfp206-exon $5 \mathrm{CpGi}$ is one of neural development and differentiation markers in mouse brain, confirming it was a DS-DMR in mouse neuronal development, and may regulate Zfp206 expression. Our previous reports indicated that the region was also a testis-specific DMR. This supports the previously reported results that Zfp206 maintained undifferentiated state of ES cells. Therefore ZFP206 may be differentially expressed in testis/germ cells and may be in a category of cancer/testis antigen, some of which are regulated by epigenetic factors, such as DNA methylation (24).

Neuroblastoma is related with neural development factors, such as NGF-dependent tyrosine kinase receptor TrkA activation, which relates to differentiation in normal and neoplastic neuronal cells. High expression of TrkA is associated with favorable outcome in neuroblastoma $(10,25,26)$. Although there were several prognostic factors of favorable outcomes in neuroblastomas, hypermehtylation level of the 5'-end of ZNF206-exon 5 CpGi was significantly correlated with ZFP206 expression and favorable outcome suggesting that ZFP206 and the DNA methylation could be prognostic biomarkers in neuroblastoma.

Mehylation of $\mathrm{CpG}$ sites at exonic region, which is not a promoter region, may be linked to epigenetic remodeling of genomic DNA structure. One is the CCCTC binding factor (CTCF), which is highly conserved in higher eukaryotes. CTCF binds to CTCF-binding sites, which are often regulated by DNA methylation. CTCF-binding sites are located at $45 \%$ intergenic, 7\% 5' untranslated region (UTR), 3\% exonic, 29\% intronic and $20 \%$ within $2.5 \mathrm{~kb}$ of promoters. H19 DMR is one of CTCF-binding sites and its methylation level is related to epigenetic remodeling, which is co-localized with cohesion. Aberrant regulation of CTCF expression is associated with occurrence of cancers, such as colorectal carcinoma (27-29). Another alternative regulation of gene expression by methylation of $\mathrm{CpG}$ site at intragenic regions is related with $3^{\prime} \mathrm{UTR}$ binding proteins, such as AU-rich element binding factor 1 (AUF1) and Hu antigen R (HUR). AUF1 and HUR are mRNA stability proteins and bind to 3'UTR. Their binding affinity is regulated by DNA methylation. It is reported that AUF1 and $\mathrm{HuR} /$ methyl-HuR regulate MAT expression and play a role in liver proliferation, differentiation and carcinogenesis $(30,31)$. The CpG sites at CpGi in ZNF206-exon 5 are located on the 5'end of exon 5 to $3^{\prime}$ UTR, however, its relationship with CTCF and $3^{\prime}$ UTR binding proteins remains elusive. Although further analysis is needed, it is possible that these regions play a role in regulation of the ZNF206 expression.

This is the first report indicating that DNA methylation level of Zfp206-exon 5 CpGi is associated with Zfp206 expression and aberrantly methylated in neuroblastomas. Hyper-methylation of the region might be one of the prognostic factors in this tumor. Although regulation mechanism of ZNF206 expression and its function remain elusive, our present data strongly suggested that this gene could be involved in neural differentiation and human neuroblastomagenesis.

\section{Acknowledgments}

This work was supported by a Nihon University Multidisciplinary Research Grant for 2006, 2007 (to Hiroki Nagase), the Academic Frontier Project for 2006 Project for Private Universities, a matching fund subsidy from MEXT (to Hiroki Nagase), and a grant-in-aid from the Ministry of Education, Science, Sports and Culture of Japan (to Taro Ikeda, grant no. 20592092).

\section{References}

1. Geiman TM and Muegge K: DNA methylation in early development. Mol Reprod Dev 77: 105-113, 2010.

2. Bird A: DNA methylation patterns and epigenetic memory. Genes Dev 16: 6-21, 2002.

3. Eckhardt F, Lewin J, Cortese R, Rakyan VK, Attwood J, Burger M, Burton J, Cox TV, Davies R, Down TA, Haefliger C, Horton R, Howe K, Jackson DK, Kunde J, Koenig C, Liddle J, Niblett D, Otto T, Pettett R, Seemann S, Thompson C, WestT, Rogers J, Olek A, Berlin K and Beck S: DNA methylation profiling of human chromosomes 6, 20 and 22. Nat Genet 38: 1378-1385, 2006.

4. Schilling E and Rehli M: Global, comparative analysis of tissuespecific promoter CpG methylation. Genomics 90: 314-323, 2007.

5. Liang P, Song F, Ghosh S, Morien E, Qin M, Mahmood S, Fujiwara K, Igarashi J, Nagase $\mathrm{H}$ and Held WA: Genome-wide survey reveals dynamic widespread tissue-specific changes in DNA methylation during development. BMC Genomics (In press).

6. Sharma S, Kelly TK and Jones PA: Epigenetics in cancer. Carcinogenesis 31: 27-36, 2009.

7. Avraham A, Sandbank J, Yarom N, Shalom A, Karni T, Pappo I, Sella A, Fich A, Walfisch S, Gheber L and Evron E : A similar cell-specific pattern of HOXA methylation in normal and cancer tissues. Epigenetics 5: 41-46, 2010.

8. Maris JM, Hogarty MD, Bagatell R and Cohn SL: Neuroblastoma. Lancet 369: 2106-2120, 2007

9. Matthay KK, Villablanca JG, Seeger RC, Stram DO, Harris RE, Ramsay NK, Swift P, Shimada H, Black CT, Brodeur GM, Gerbing RB and Reynolds CP: Treatment of high-risk neuroblastoma with intensive chemotherapy, radiotherapy, autologous bone marrow transplantation, and 13-cis-retinoic acid. Children's Cancer Group. N Engl J Med 341: 1165-1173, 1999.

10. Brodeur GM: Neuroblastoma: biological insights into a clinical enigma. Nat Rev Cancer 3: 203-216, 2003. 
11. Song F, Mahmood S, Ghosh S, Liang P, Smiraglia DJ, Nagase H and Held WA: Tissue specific differentially methylated regions (TDMR): Changes in DNA methylation during development. Genomics 93: 130-139, 2009.

12. Ehrich M, Nelson MR, Stanssens P, Zabeau M, Liloglou T, Xinarianos G, Cantor CR, Field JK and van den Boom D: Quantitative high-throughput analysis of DNA methylation patterns by base-specific cleavage and mass spectrometry. Proc Natl Acad Sci USA 102: 15785-15790, 2005.

13. Li LC and Dahiya R: MethPrimer: designing primers for methylation PCRs. Bioinformatics 18: 1427-1431, 2002.

14. Fatemi M,Pao MM,Jeong S, Gal-Yam EN,EggerG, Weinberger DJ and Jones PA: Footprinting of mammalian promoters: use of a CpG DNA methyltransferase revealing nucleosome positions at a single molecule level. Nucleic Acids Res 33: e176, 2005.

15. Akoben AK: Understanding diagnostic tests 3 : Receiver operating characteristic curves. Acta Paediatr 96: 644-647, 2007.

16. Egger G, Liang G, Aparicio A and Jones PA: Epigenetics in human disease and prospects for epigenetic therapy. Nature 27 457-463, 2004.

17. Laird PW: Cancer epigenetics. Hum Mol Genet 15: 65-76, 2005.

18. Gonzalez-Gomez P, Bello MJ, Lomas J, Arjona D, Alonso ME, Amiñoso C,Lopez-Marin I, Anselmo NP, Sarasa JL, Gutierrez M, Casartelli $\mathrm{C}$ and Rey JA: Aberrant methylation of multiple genes in neuroblastic tumours. relationship with MYCN amplification and allelic status at 1p. Eur J Cancer 39: 1478-1485, 2003.

19. Banelli B, Vinci AD, Gelvi I, Casciano I, Allemanni G, Bonassi S and Romani M: DNA methylation in neuroblastic tumors. Cancer Lett 228: 37-41, 2005

20. Huges TR: Zfp206 regulates ES cell gene expression and differentiation. Nucreic Acid Res 34: 4780-4790, 2006.

21. Wang ZX, Kueh JL, Teh CH, Rossbach M, Lim L, Li P, Wong KY, Lufkin T, Robson P and Stanton LW: Zfp206 is a transcriptinon factor that controls pluripotency of embryonic stem cells. Stem Cells 25: 2173-2182, 2007.

22. Wang ZX, Teh CH, Kueh JL, Lufkin T, Robson P and Stanton LW: Oct 4 and Sox 4 directly regulate expression of another pulipotency transcription factor, Zfp206, in embryonic stem cells. J Bio Chem 282: 12822-12830, 2007.
23. Yu HB, Kunarso G, Hong FH and Stanton LW: Zfp206, Oct4 and Sox 2 are integrated components of a transcriptional regulatory network in embryonic stem cells. J Bio Chem 284: 31327-31335, 2009.

24. Maio M, Coral S, Fratta E, Altomonte M and Sigalotti L: Epigenetic targets for immune intervention in human malignancies. Oncogene 22: 6484-6488, 2003.

25. Nakagawara A, Arima-Nakagawara M, Scavarda NJ, Azar CG, Cantor AB and Brodeur GM: Association between high levels of expression of the TRK gene and favorable outcome in human neuroblastoma. N Engl J Med 328: 847-854, 1993.

26. Schramm A, Schulte JH, Astrahantseff K, Apostolov O, Limpt V, Sieverts H, Kuhfittig-Kulle S, Pfeiffer P, Versteeg R and Eggert A: Biological effects of TrkA and TrkB receptor signaling in neuroblastoma. Cancer Lett 228: 143-153, 2005.

27. Ishihara K, Oshimura M and Nakao M: CTCF-dependent chromatin insulator is linked to epigenetic remodeling. Mol Cell 23: 733-742, 2006

28. Wendt KS, Yoshida K, Itoh T, Bando M, Koch B, Schirghuber E, Tsutsumi S, Nagae G, Ishihara K, Mishiro T, Yahata K, Imamoto F, Aburatani H, Nakao M, Imamoto N, Maeshima K, Shirahige K and Peters JM: Cohesin mediates transcriptional insulation by CCCTC-binding factor. Nature 451: 796-801, 2008.

29. Phillips JE and Corces VG: CTCF: master weaver of the genome. Cell 137: 1194-1211, 2009.

30. Torrisani J, Unterberger A, Tendulkar SR, Shikimi K and Szyf M: AUF1 cell cycle variations define genomic DNA methylation by regulation of DNMT1 mRNA stability. Mol Cell Biol 27: 395-410, 2007.

31. Vázquez-Chantada M, Fernández-Ramos D, Embade N, Martínez-Lopez N, Varela-Rey M, Woodhoo A, Luka Z, Wagner C, Anglim PP, Finnell RH, Caballería J, LairdOffringa IA, Gorospe M, Lu SC, Mato JM, Martínez-Chantar ML: $\mathrm{HuR} / \mathrm{methyl-HuR}$ and AUF1 regulate the MAT expressed during liver proliferation, differentiation, and carcinogenesis. Gastroenterology 138: 1943-1953, 2010. 